\title{
Future Groundbased High-Resolution IR Spectrometers
}

\author{
Alan Moorwood \\ European Southern Observatory, Garching, D-85748, Germany
}

\begin{abstract}
Plans for future high-resolution infrared spectrometers on large groundbased telescopes, their capabilities and science goals are briefly reviewed.
\end{abstract}

\section{Introduction}

High spectral resolution astronomical observations in the infrared were pioneered by Pierre Connes in the 1960s using Fourier Transform spectrometers, which could yield resolving powers $\simeq 10^{6}$ but whose sensitivity was limited by the poor, single pixel detectors then available. This restricted initial studies mostly to the brighter planets - extended later to the brighter stars, for example, using the very successful FTS operated for many years at the KPNO.

More recently, the development of highly sensitive, large format infrared array detectors has stimulated increasing interest in the development of cryogenic echelle spectrometers, which are currently being limited to somewhat lower resolving powers of $\simeq 10^{5}$ for practical reasons but are many orders of magnitude more sensitive than the early Fourier spectrometers. In the 1-5 $\mu \mathrm{m}$ range, Phoenix, also developed at KPNO for use on the $4 \mathrm{~m}$ Mayall telescope and now installed on the $8 \mathrm{~m}$ Gemini South telescope (http://www.gemini.edu/sciops/ instruments/phoenix/phoenixIndex.html) has pioneered this approach. A pioneering instrument in the $8-20 \mu \mathrm{m}$ range is Michelle, developed by the UKATC in Edinburgh for UKIRT but now on Gemini North (http://www.gemini.edu/ sciops/instruments/michelle/MichIndex.html). The increase in science objectives possible with such instruments is illustrated in the next section, and characteristics of the new high-resolution spectrometers CRIRES and VISIR, to be installed on the ESO VLT in the near future, are given in section 3.

\section{Infrared Astronomy at High Spectral Resolution}

\section{Table 1. Science Objectives}

- Planetary atmospheres

- Exoplanets (radial velocity reflex motion searches; direct detection)

- Stars (atomic and molecular abundances, winds, pulsations, magnetic fields, disks, outflows)

- Interstellar Medium (chemistry and kinematics)

- Galaxies/quasars (nuclear kinematics, intergalactic gas) 


\section{IR Spectrometer Developments}

\subsection{CRIRES}

CRIRES (http://www.eso.org/instruments/crires) is being developed for one of the VLT $8 \mathrm{~m}$ telescopes by ESO at its headquarters in Garching under the leadership of the author.

\section{Table 2. CRIRES Main Characteristics}

- Located at Nasmyth focus of one of the $8 \mathrm{~m}$ VLTs

- Wavelength range 1-5 $\mu \mathrm{m}$

- $\mathrm{R}=10^{5}$ with $0.1^{\prime \prime}$ pixels; $50^{\prime \prime}$ long slit

- Adaptive optics feed to maximize SNR and spatial resolution

- Slit viewing camera for IR acquisition

- Echelle grating (R2) and prism pre-disperser

- 4096x512 pixel InSb detector mosaic

- Polarimetry (including circular) with Fresnel rhomb and Wollaston prism

- Calibration unit including gas cells for accurate radial velocities

- Limiting magnitudes $\simeq 17(\mathrm{~J})$ to $11(\mathrm{M})$ in $1 \mathrm{hr}$

- Installation in early 2005

\subsection{VISIR}

VISIR ( http://www.eso.org/instruments/visir) is a multimode imager/spectrometer designed to operate in the 10 and $20 \mu \mathrm{m}$ atmospheric windows. It is being developed for the ESO VLT by the Service d'Astrophysique, CEA, Saclay and NFRA/ASTRON, Dwingeloo, The Netherlands under Pierre - Olivier Lagage at Principal Investigator.

\section{Table 3. VISIR Main Characteristics}

- Cassegrain focus of the VLT

- Wavelength Range 5-28 $\mu \mathrm{m}$

- Duo echelle grating + cross disperser grisms

- 256x256 pixel As:Si array

- $\mathrm{R} \simeq 25000$ at diffraction limit

- Installation on VLT March 2004 